\title{
Increased uptake of fluorine-18 fluoro-2-deoxy-D- glucose (F18-FDG) in the spleen: A novel marker for severe Kikuchi-Fujimoto disease
}

Hye Seong

Severance Hospital https://orcid.org/0000-0002-5633-7214

\section{Yong Hyu Jeong}

Severance Hospital

Lee Woon ji

Severance Hospital

Jun Hyoung Kim

Severance Hospital

Jung Ho Kim

Severance Hospital

Jin Young Ahn

Severance Hospital

Su Jin Jeong

Severance Hospital

Jun Yong Choi

Severance Hospital

Yoon Soo Park

Severance Hospital

Joon Sup Yeom

Severance Hospital

\section{Young Goo Song}

Severance Hospital

Arthur Cho

Severance Hospital

Nam Su Ku ( $\nabla$ smileboy9@yuhs.ac )

https://orcid.org/0000-0002-9717-4327

Original research

Keywords: Kikuchi-Fujimoto disease (KFD), Fluorine-18 Fluoro-2-deoxy-D-glucose positron emission tomography/computed tomography (F18-FDG PET/CT), standardized uptake values (SUV), metabolic 
tumor volume (MTV), total lesion glycolysis (TLG)

Posted Date: June 4th, 2020

DOI: https://doi.org/10.21203/rs.3.rs-32657/v1

License: (c) (1) This work is licensed under a Creative Commons Attribution 4.0 International License. Read Full License 
4 Hye Seong ${ }^{1,2 *}$, Yong Hyu Jeong ${ }^{3 *}$, Woon Ji Lee ${ }^{1}$, Jun Hyoung Kim ${ }^{1}$, Jung Ho Kim ${ }^{1}$, Jin Young Ahn ${ }^{1}$, 5 Su Jin Jeong ${ }^{1}$, Jun Yong Choi ${ }^{1}$, Yoon Soo Park ${ }^{1}$, Joon Sup Yeom ${ }^{1}$, Young Goo Song ${ }^{1}$, Arthur Cho ${ }^{3 \#,}$ 6 and $\mathrm{Nam} \mathrm{Su} \mathrm{Ku}{ }^{1 \#}$

$7 \quad{ }^{1}$ Department of Internal Medicine and AIDS Research Institute, Yonsei University College of Medicine, 8 Seoul, Republic of Korea

\section{Increased uptake of fluorine-18 fluoro-2-deoxy-D-glucose in the spleen: A} novel marker for severe Kikuchi-Fujimoto disease

${ }^{2}$ Department of Internal Medicine, Korea University Guro Hospital, Seoul, Republic of Korea

${ }^{3}$ Department of Nuclear Medicine, Severance Hospital, Yonsei University College of Medicine, Seoul, Republic of Korea

*Hye Seong and Yong Hyu Jeong equally contributed to this study.

\section{${ }^{\#}$ Co-corresponding authors}

Nam Su Ku, M.D., Ph.D.

Department of Internal Medicine, Yonsei University College of Medicine, 50-1 Yonsei-ro,

Seodaemoon-Gu, Seoul, Korea, 120-752

Phone: 82-2-2228-2277

Fax: 82-2-393-6884

E-mail: smileboy9@yuhs.ac

Arthur Cho, M.D., Ph.D.

Department of Nuclear Medicine, Yonsei University College of Medicine, 50-1 Yonsei-ro, 
Phone: 82-2-2228-2349

Fax: 82-2-393-6884

E-mail: artycho@yuhs.ac

\section{Abstract}

Background: Kikuchi-Fujimoto disease (KFD), known as histiocytic necrotizing lymphadenitis, is an Asian-endemic disease of unknown etiology. Although KFD is usually self-limiting and benign, patients with prolonged systemic symptoms often have frequent hospital visits, long admission durations, and missed workdays. Immune-modulating drugs are used to shorten the clinical course in severe KFD. However, as there are no established severity markers, administration of these drugs is often arbitrary or delayed. We aimed to investigate the role of fluorine-18 fluoro-2-deoxy-D-glucose (F18-FDG) positron emission tomography/computed tomography (F18-FDG PET/CT) in KFD and to evaluate its performance as a disease severity marker. We retrospectively reviewed medical records of 31 adult

Results: The median value of the mean splenic standardized uptake value ( $\left.\mathrm{SUV}_{\text {mean }}\right)$ was higher in the 23 patients with severe KFD ( $1.79 \pm 0.99$ vs. $2.38 \pm 1.18, p=0.058)$. Patients with severe KFD presented with more systemically activated volume and glycolytic activity than those with mild KFD (total lesion myalgia (odds ratio [OR], 0.035; 95\% confidence interval [CI], 0.001-0.792; $p=0.035$ ), total lymph 
47 node $\mathrm{SUV}_{\max }$ (cutoff, 9.27) (OR, 24.734; 95\% CI, 1.323-462.407; $p=0.032$ ), and spleen $\mathrm{SUV}_{\text {mean }}$ (cutoff, 48 1.79) (OR, 37.770; 95\% CI, 1.769-806.583; $p=0.020)$ were significantly associated with severe KFD.

49 Conclusions: We suggest that ${ }^{18} \mathrm{~F}-\mathrm{FDG}$ PET/CT can be a useful tool for clinical workup in the 50 predicting clinical course of KFD as a complement to laboratory and clinical findings to establish the 51 severity.

52 Keywords: Kikuchi-Fujimoto disease (KFD), Fluorine-18 Fluoro-2-deoxy-D-glucose positron emission 53 tomography/computed tomography (F18-FDG PET/CT), standardized uptake values (SUV), metabolic tumor volume (MTV), 54 total lesion glycolysis (TLG) 
Kikuchi-Fujimoto disease (KFD), also known as histiocytic necrotizing lymphadenitis, is an Asianendemic disease of unknown etiology [1-3]. It usually occurs in young adult women and is most commonly characterized by cervical lymphadenopathy and fever $[4,5]$. KFD presents with various clinical features, from no systemic symptoms to significant symptoms like night sweats, myalgia, weight loss, arthralgia, and hemophagocytic lymphohistiocytosis (HLH) [6-9].

Although KFD is usually benign and self-limiting, patients with prolonged systemic symptoms are plagued with frequent hospital visits, long durations of admission, or missed workdays [10]. Immunemodulating drugs such as high-dose corticosteroids or intravenous immunoglobulin have been used to treat patients with severe KFD, which helps shorten the clinical course of the disease [11, 12]. However, because there are no established markers for KFD severity, determining treatment options for KFD is dependent only on the clinician's discretion, which may result in delayed treatment or a prolonged symptom duration.

Fluorine-18 fluoro-2-deoxy-D-glucose (F18-FDG) positron emission tomography/computed tomography (PET/CT) can be used to evaluate glucose utilization in multiple organs. Although F18FDG has been predominately used to evaluate cancer metabolism, F18-FDG PET/CT has found clinical use in localizing inflammatory foci and infectious diseases such as tuberculosis, Q fever, infective endocarditis, vascular graft infection, chronic active Epstein-Barr virus infection, invasive fungal infection, and surgical site infection [13-17]. Another advantage of F18-FDG PET/CT is that PET metrics such as standardized uptake value (SUV), metabolic tumor volume (MTV), and total lesion glycolysis (TLG) allows for an evaluation of the severity and quantification of glycolysis in multiple organs, which are useful as prognosis prediction parameters for survival in patients affected by solid tumors [18].

The spleen is an important immune organ in both innate and adaptive immune responses and in regulating immune homeostasis [19]. Studies have shown diffuse increased splenic F18-FDG uptake in lymphoma, infection, tuberculosis, and autoimmune diseases [20-23]. Moreover, recent findings 
suggest that a diffuse increased FDG uptake was observed not only in lymph nodes but also in the spleen in patients with KFD [24, 25]. However, the relationship between abnormal F18-FDG uptake in patients with KFD and the disease severity has not yet been described.

We hypothesized that glucose metabolism in the spleen and pathologic lymph nodes varies according to KFD severity because of the presence of systemic inflammation. Hence, we investigated the uptake of F18-FDG in the spleen and lymph nodes in patients with KFD and evaluated its performance as a disease severity parameter.

\section{Methods}

\section{Patients selection}

We retrospectively reviewed the electronic medical records of 31 adult patients who had pathologically confirmed KFD and who underwent F18-FDG PET/CT between November 2007 and April 2018 at a tertiary care referral hospital located in Seoul, Korea. Data collected included age, sex, clinical manifestations, laboratory test values, histologic findings, treatment methods and durations, fever duration, and outcomes such as relapse.

All procedures performed in human studies were in accordance with the ethical standards of the institutional and/or national research committee and with the 1964 Helsinki Declaration and its later amendments or comparable ethical standards. This study was approved by the Institutional Review Board of Yonsei University Health System Clinical Trial Center (4-2019-0977). Since the study was retrospective and the study subjects were anonymized, the Institutional Review Board waived the requirement for patients' written consent.

\section{Definition of severe KFD}

For assessment of disease severity, we established the presence of severe KFD based on the clinical manifestations of advanced KFD disease [3, 10, 26]. Severe KFD was defined as KFD with at least one 
of the following manifestations; encephalitis, peripheral neuropathy, HLH, long fever duration ( $>7$ days), and leukopenia $(<4000 / \mu \mathrm{L})$.

\section{Imaging technique}

All patients fasted for at least 6 hours before the PET/CT scans were taken. Serum glucose levels (preferably $<150 \mathrm{mg} / \mathrm{dL}$ ) were measured followed by intravenous administration of $5.5 \mathrm{MBq} / \mathrm{kg} \mathrm{F} 18$ FDG (with a maximum of $400 \mathrm{MBq}$ ). PET and combined low-dose CT scans were performed with commercial PET/CT scanners (Discovery STE, Discovery D600, Discovery D710 [GE Healthcare], or Biograph TruePoint40 [Siemens Medical Systems]) after 1 hour. The PET scan was performed with an acquisition time of 2 min per bed position in the 3-dimensional mode. PET data were reconstructed iteratively using an ordered subset expectation maximization algorithm with the low-dose CT datasets for attenuation correction.

\section{Imaging analysis}

For semi-quantitative and volumetric analysis, various metabolic PET parameters were measured including the maximum standardized uptake value $\left(\mathrm{SUV}_{\max }\right), \mathrm{MTV}$, and TLG in the lymph nodes as well as the mean standardized uptake values ( $\left.\mathrm{SUV}_{\text {mean }}\right)$ and TLG in the spleen using a commercially available imaging software (MIM Software, Cleveland, Ohio). All PET/CT images were read by two experienced nuclear medicine physicians. For background activity, a spherical volume of interest (VOI) with a diameter of $30 \mathrm{~mm}$ was drawn at the inferior right lobe of the liver, excluding the main ducts and vessels. The $\mathrm{SUV}_{\text {mean }}$ of that VOI was adopted as a threshold value to determine the boundaries of the pathologic lymph nodes in each PET/CT study. After SUV thresholding, FDG-avid regions were automatically segmented using the isocontour threshold method. The $\mathrm{SUV}_{\max }$ of the total lymph nodes was defined as the highest metabolic foci $\left(\mathrm{SUV}_{\max }\right)$ in all the metabolically active lymph nodes in the body. The total MTV of lymph nodes was defined as the sum of the MTVs of all individual focal lesions identified in the analysis. The TLG of each focal lesion was calculated by multiplying the SUV $\mathrm{mean}_{\text {and }}$ voxel number of that lesion. The total lymph node TLG of each patient was defined as the sum of the TLGs for all focal lesions in the analysis. The $\mathrm{SUV}_{\text {mean }}$ and TLG of the spleen were identified by 
manually drawing regions of interest on each slice of the attenuation-corrected axial PET images (Figure 1).

\section{Statistical Analysis}

Descriptive statistics for continuous variables are represented as medians \pm interquartile range (IQR), and categorical variables are shown as numbers (percentage). The Mann-Whitney U test was performed to analyze differences between the mild and severe groups, while the $\chi^{2}$ test or Fisher's exact test was performed on categorical data using SPSS 25.0 (SPSS Inc., Chicago, IL, USA). To determine independent predictors of severity in the KFD group, we performed a multivariable analysis with a logistic regression model including risk factors associated with a P-value of less than 0.05 in the univariate analysis. Differences were considered to be statistically significant at a 2-sided P-value of less than 0.05 .

A receiver operating characteristic (ROC) analysis was used to describe the relationship between FDG uptake and disease severity. MedCalc software (version 19.1, Ostend, Belgium) was used for analyzing the data. Results of F18-FDG PET/CT in the severe group were compared with those in the mild group to assess the diagnostic performance of F18-FDG PET/CT in evaluating the degree of severity of KFD. The diagnostic performance was expressed in terms of sensitivity, specificity, Youden index, positive predictive value (PPV), and negative predictive value (NPV).

\section{Results}

\section{Patient's characteristics}

The baseline characteristics of the 31 patients with KFD who underwent F18-FDG PET/CT are summarized in Table 1. All patients had been confirmed with KFD based on the pathological findings of a biopsy. The median age was 27.5 years (IQR, 28 years), and 13 patients were men (41.9\%). The most commonly affected site of lymphadenopathy was the neck $(\mathrm{n}=18 ; 58.1 \%)$ followed by the axilla $(\mathrm{n}=7 ; 22.6 \%)$. In terms of systemic symptoms, almost every patient in our study presented with a fever $(\mathrm{n}=30 ; 96.8 \%)$. Among the study patients, 7 (25.8\%) patients were categorized into the mild group and 
$24(74.2 \%)$ into the severe KFD group. There were significant differences in age (38.0 \pm 29 vs. $26.0 \pm 18$ years, $p=0.038)$ and lactate dehydrogenase (LDH) levels ( $310.5 \pm 298$ vs. $612.5 \pm 672, p=0.033)$. However, there were no significant differences in $\operatorname{sex}(57.1 \%$ vs. $37.5 \%, p=0.354)$, sites of lymphadenopathy, and systemic symptoms between the mild and severe groups.

$\{$ Insert Table 1 here $\}$

\section{Comparison of PET/CT parameters according to the severity of KFD}

We investigated the locations, metabolic activity, and size of hypermetabolic lymph nodes on F18-FDG PET/CT images. The findings from the F18-FDG PET/CT examinations are presented in Table 1. We identified hypermetabolic lymph nodes in 31 patients with maximum standard uptake values ( $\left.\mathrm{SUV}_{\max }\right)$ from the neck, axilla, mediastinum, and the abdominopelvic area. Hypermetabolic lymph nodes were observed in the necks of 18 patients, axillas of 7 , mediastinums of 4 , and abdomens and pelvis of 2 patients. The sum of the median $\mathrm{SUV}_{\max }, \mathrm{MTV}$, and TLG of F18-FDG uptake in affected lymph nodes were $10.65 \pm 7.64,97.95 \pm 112.29$, and $398.73 \pm 464.08$, respectively. The F18-FDG uptake in the spleen (SUV ${ }_{\text {mean }}, 2.19 \pm 1.14$; TLG, 539.36 \pm 395.69$)$ was calculated for all patients.

The median F18-FDG PET/CT parameters involving the lymph nodes, liver, and spleen were identified in the mild and severe groups. The spleen $\mathrm{SUV}_{\text {mean }}$ was higher in patients with severe KFD $(1.79 \pm 0.99$ vs. $2.38 \pm 1.18, p=0.058)$. The median values of total lymph node $\operatorname{SUV}_{\max }(8.19 \pm 7.10$ vs. $11.68 \pm 7.33$, $p=0.214)$, MTV (61.85 \pm 118.32 vs. $99.36 \pm 120.21, p=0.104)$, and total lymph node TLG $(201.57 \pm 363.45$ vs. $473.52 \pm 504.44, p=0.024$ ) were higher in the severe group than in the mild group, indicating a higher F18-FDG uptake in the severe group.

\section{Diagnostic performance of F18-FDG PET/CT in the prediction of KFD severity}

Prediction of severity was made by analyzing the area under the curve (AUC) of the ROC (Figure 2), and the corresponding statistics are shown in Table 2. Using the definition of severe KFD as a diagnostic criterion to separate the severe group from the mild group, a ROC curve analysis determined the most sensitive and specific cutoff values for total lymph node TLG, spleen $\mathrm{SUV}_{\text {mean, }}$, total lymph node MTV, 
Table 2. Diagnostic performance of PET-CT for severity of Kikuchi-Fujimoto disease

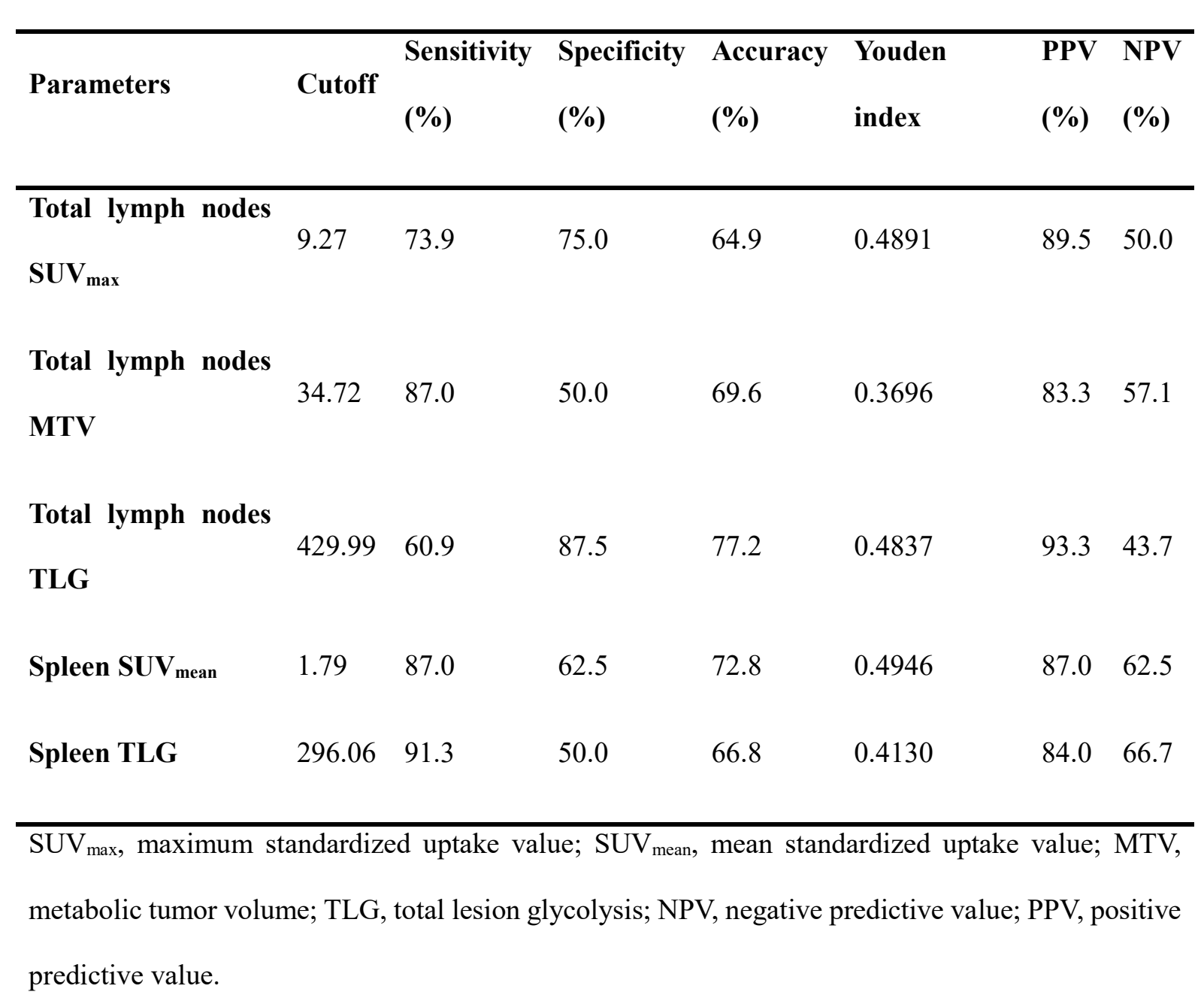

spleen TLG, and total lymph node $\mathrm{SUV}_{\max }$ as 429.99, 1.79, 34.72, 296.06, and 9.27, respectively. Additionally, the calculated sensitivity, specificity, NPV, and PPV of total lymph node TLG (sensitivity, 60.9\%; specificity, 87.5\%; PPV, 93.3\%, NPV 43.7\%), spleen $\mathrm{SUV}_{\text {mean }}$ (sensitivity, 87.0\%; specificity, 62.5\%; PPV, 87.0\%, NPV 62.5\%), total lymph node MTV (sensitivity, 87.0\%; specificity, 50.0\%; PPV, 83.3\%, NPV 57.1\%) and total lymph node $\mathrm{SUV}_{\max }$ (sensitivity, $73.9 \%$; specificity, $75.0 \%$; PPV, 89.5\%, NPV 50.0\%) at the cutoff value are presented in Table 2. Among these parameters, total lymph node TLG $(77.2 \%)$ and spleen $\operatorname{SUV}_{\text {mean }}(72.8 \%)$ were found to be more accurate than the other parameters. 
We evaluated the predictive factors for severe KFD. A univariate and multivariable analysis of severity in KFD was performed and is presented in Table 3. In the univariate logistic regression, myalgia (odds ratio $[\mathrm{OR}], 0.150 ; 95 \%$ confidence interval $[\mathrm{CI}], 0.024-0.946 ; p=0.044)$, total lymph node $\mathrm{SUV}_{\max }$ (cutoff 9.27) (OR, 8.500; 95\% CI, 1.335-54.127; $p=0.023)$, total lymph node MTV (cutoff, 34.72) (OR, 6.667; 95\% CI, 1.057-42.065; $p=0.044)$, total lymph node TLG (cutoff, 429.99) (OR, 10.889; 95\% CI, 1.140-103.977; $p=0.038)$, spleen $\mathrm{SUV}_{\text {mean }}$ (cutoff, 1.79) (OR, 11.111; 95\% CI, 1.701-72.564; $p=0.012$ ) and spleen TLG (cutoff, 296.06) (OR, 10.500; 95\% CI, 1.412-78.059; $p=0.022$ ) were statistically significant. The multivariate logistic regression model showed that the myalgia $(\mathrm{OR}, 0.035 ; 95 \% \mathrm{CI}$, 0.001-0.792; $p=0.035)$, total lymph node $\mathrm{SUV}_{\max }$ (cutoff, 9.27) (OR, 24.734; 95\% CI, 1.323-462.407; $p=0.032)$, and spleen $\mathrm{SUV}_{\text {mean }}$ (cutoff, 1.79) (OR, 37.770; 95\% CI, 1.769-806.583; $\left.p=0.020\right)$ were significantly associated with severe KFD.

\{Insert Table 3 here $\}$

\section{Discussion}

The clinical utility of F18-FDG PET/CT includes various inflammatory and infectious diseases and benign disorders [27]. Due to the advantages of F18-FDG PET/CT in the systematic evaluation of fever of unknown origin [28], F18-FDG has often been used for the diagnostic workup of KFD. Alshammari et al. reported that F18-FDG uptake can be detected not only in the generalized lymph nodes but also in the spleen in patients with KFD [24]. Another study reported that the spleen showed increased F18FDG uptake in patients with febrile autoimmune disease and is associated with an increased risk of allcause in-hospital mortality [22].

In this study, myalgia was found to be correlated with mild KFD. This may be because patients with mild KFD often present with myalgia at the time of diagnosis. Furthermore, patients presenting with myalgia as a systemic symptom are usually evaluated for the disease earlier than those who do not present with myalgia. We investigated the values of F18-FDG PET/CT in patients with severe KFD to determine whether they can be used as predictive factors for disease severity. Among the various F18FDG PET/CT parameters, total lymph node $\mathrm{SUV}_{\max }$ and spleen $\mathrm{SUV}_{\text {mean }}$ were significantly associated 
with severe KFD. F18-FDG uptake was significantly higher not only in the affected lymph nodes but also in the spleen in severe KFD. In a multivariate logistic regression analysis, the total lymph node $\mathrm{SUV}_{\max }$ with a cutoff value higher than 9.27 and spleen $\mathrm{SUV}_{\text {mean }}$ with a cutoff value higher than 1.79 were independent predictors of KFD severity. Increased total lymph node $\mathrm{SUV}_{\max }$ and spleen $\mathrm{SUV}_{\text {mean }}$ might be useful for predicting the disease course when clinical or laboratory data are not available or are not confirmed.

The spleen is the largest lymphoid organ in the human body that regulates blood flow and filters microorganisms [19]. As a specialized immune organ, the spleen has various functions such as clearance of microorganisms, site of development for lymphocytes (both $\mathrm{T}$ and B), release of immunoglobulins, and production of immune mediators [29]. Generally, F18-FDG uptake is related to tissue metabolism, which may explain why an increased F18-FDG uptake in the spleen may reflect increased glucose consumption in the spleen in the event of an infection [21]. A recent study demonstrated that current inflammation could result in diffuse splenic F18-FDG uptake [30]. Therefore, we could presume that increased diffuse F18-FDG uptake in the spleen can be seen in many inflammatory diseases reflecting the activation of the immune system in the spleen. This relationship between splenic glucose metabolism and inflammation may help explain our results.

There are several limitations to this study. First, it was a retrospective study. Second, our study population was small due to the low prevalence of KFD and the high cost of F18-FDG PET/CT. Finally, we defined severe KFD arbitrarily. Since the severity criteria of KFD have not been previously defined, we defined severe KFD based on previous reports and clinical experience. Despite these limitations, our study is the first, to the best of our knowledge, to evaluate the potential association between F18FDG PET/CT parameters and KFD severity.

\section{Conclusion}

Our study suggests that F18-FDG PET/CT can be a useful tool in evaluating patients with KFD and 
establishing disease severity as a complement to laboratory and clinical findings. Further studies with

243 larger populations are warranted to validate our results regarding the role of F18-FDG PET/CT in

244 determining KFD severity.

245

246 List of abbreviations

$247 \quad$ AUC

Area under the curve

$248 \quad \mathrm{KFD}$

Kikuchi-Fujimoto disease

249

MIP

Maximum intensity projection

250 MTV

Metabolic tumor volume

251

NPV

Negative predictive value

252

PET

Positron emission tomography/computed tomography

253

PPV

Positive predictive value

254 ROC

Receiver operating characteristic

255

SUV

Standardized uptake value

256 TLG

Total lesion glycolysis

$257 \quad$ VOI

Volume of interest

Declarations

Ethics approval and consent to participate

261 All procedures performed in studies were in accordance with the ethical standards of the institutional and/or national research committee and with the 1964 Helsinki Declaration and its later amendments or comparable ethical standards. 
This study was approved by the Institutional Review Board of Yonsei University Health System Clinical Trial Center (4-2019-0977). Since the study was retrospective and the study subjects were anonymized, the IRB waived the requirement for patients' written consent.

\section{Consent for publication}

Not applicable.

\section{Availability of data and material}

The datasets used and/or analyzed during the current study are available from the corresponding author on reasonable request.

\section{Competing interests}

The authors declare that they have no conflict of interest. None of the authors have affiliations that present financial or non-financial competing interests for this work.

\section{Funding}

No funding was received for this study.

\section{Authors' contributions}

All authors participated either to the following: (1) study conception and design or analysis and interpretation of the data, or both (HS, YJ, WJ, JK, JK, JA, SJ, JC, YP, JY, YS, NK, AC); (2) the drafting of the manuscript or its critical revision for important intellectual content (HS, YJ, NK, AC); or (3) final approval of the submitted manuscript (NK, AC). The author(s) read and approved the final manuscript.

\section{Acknowledgements}

Not applicable. 


\section{References}

1. Chen CK, Low Y, Akhilesh M, Jacobsen AS. Kikuchi disease in Asian children. J Paediatr Child Health. 2006;42(3):104-7.

2. Seo JH, Shim HS, Park JJ, Jeon SY, Kim JP, Ahn SK, et al. A clinical study of histiocytic necrotizing lymphadenitis (Kikuchi's disease) in children. Int J Pediatr Otorhinolaryngol. 2008;72(11):1637-42.

3. Lin DY, Villegas MS, Tan PL, Wang S, Shek LP. Severe Kikuchi's disease responsive to immune modulation. Singapore Med J. 2010;51(1):e18-21.

4. Dorfman RF, Berry GJ. Kikuchi's histiocytic necrotizing lymphadenitis: an analysis of 108 cases with emphasis on differential diagnosis. Semin Diagn Pathol. 1988;5(4):329-45.

5. Chuang $\mathrm{CH}$, Yan DC, Chiu CH, Huang YC, Lin PY, Chen CJ, et al. Clinical and laboratory manifestations of Kikuchi's disease in children and differences between patients with and without prolonged fever. Pediatr Infect Dis J. 2005;24(6):551-4.

6. Chen JS, Chang KC, Cheng CN, Tsai WH, Su IJ. Childhood hemophagocytic syndrome associated with Kikuchi's disease. Haematologica. 2000;85(9):998-1000.

7. Kim YM, Lee YJ, Nam SO, Park SE, Kim JY, Lee EY. Hemophagocytic syndrome associated with Kikuchi's disease. J Korean Med Sci. 2003;18(4):592-4.

8. Kim TY, Ha KS, Kim Y, Lee J, Lee K, Lee J. Characteristics of Kikuchi-Fujimoto disease in children compared with adults. Eur J Pediatr. 2014;173(1):111-6.

9. Kucukardali Y, Solmazgul E, Kunter E, Oncul O, Yildirim S, Kaplan M. Kikuchi-Fujimoto Disease: analysis of 244 cases. Clin Rheumatol. 2007;26(1):50-4.

10. Kang HM, Kim JY, Choi EH, Lee HJ, Yun KW, Lee H. Clinical Characteristics of Severe Histiocytic Necrotizing Lymphadenitis (Kikuchi-Fujimoto Disease) in Children. J Pediatr. 2016;171:208-12 e1.

11. Yoshioka K, Miyashita T, Nakamura T, Inoue T, Yamagami K. Treatment of Histiocytic Necrotizing Lymphadenitis (Kikuchi's Disease) with Prolonged Fever by a Single Course of Methylprednisolone Pulse Therapy without Maintenance Therapy: Experience with 13 Cases. Internal Medicine. 2010;49(20):2267-70.

12. Jang YJ, Park KH, Seok HJ. Management of Kikuchi's disease using glucocorticoid. J Laryngol Otol. 2000;114(9):709-11.

13. Kouijzer IJE, Kampschreur LM, Wever PC, Hoekstra C, van Kasteren MEE, de Jager-Leclercq MGL, et al. The Value of 18F-FDG PET/CT in Diagnosis and During Follow-up in 273 Patients with Chronic Q Fever. J Nucl Med. 2018;59(1):127-33.

14. Jimenez-Ballve A, Perez-Castejon MJ, Delgado-Bolton RC, Sanchez-Enrique C, Vilacosta I, Vivas D, et al. Assessment of the diagnostic accuracy of 18 F-FDG PET/CT in prosthetic 
infective endocarditis and cardiac implantable electronic device infection: comparison of different interpretation criteria. Eur J Nucl Med Mol Imaging. 2016;43(13):2401-12.

15. Sah BR, Husmann L, Mayer D, Scherrer A, Rancic Z, Puippe G, et al. Diagnostic performance of 18F-FDG-PET/CT in vascular graft infections. Eur J Vasc Endovasc Surg. 2015;49(4):45564.

16. Toriihara A, Arai A, Nakadate M, Yamamoto K, Imadome KI, Miura O, et al. FDG-PET/CT findings of chronic active Epstein-Barr virus infection. Leuk Lymphoma. 2018;59(6):1470-3.

17. Leroy-Freschini B, Treglia G, Argemi X, Bund C, Kessler R, Herbrecht R, et al. 18F-FDG $\mathrm{PET} / \mathrm{CT}$ for invasive fungal infection in immunocompromised patients. QJM. 2018;111(9):613-22.

18. Hyun SH, Choi JY, Shim YM, Kim K, Lee SJ, Cho YS, et al. Prognostic value of metabolic tumor volume measured by $18 \mathrm{~F}$-fluorodeoxyglucose positron emission tomography in patients with esophageal carcinoma. Ann Surg Oncol. 2010;17(1):115-22.

19. Mebius RE, Kraal G. Structure and function of the spleen. Nat Rev Immunol. 2005;5(8):60616.

20. Lustberg MB, Aras O, Meisenberg BR. FDG PET/CT findings in acute adult mononucleosis mimicking malignant lymphoma. Eur J Haematol. 2008;81(2):154-6.

21. Kim K, Kim SJ, Kim IJ, Kim BS, Pak K, Kim H. Diffuse increased splenic F-18 fluorodeoxyglucose uptake may be an indirect sign of acute pyogenic cause rather than tuberculous in patients with infectious spondylitis. Nucl Med Commun. 2011;32(12):1155-61.

22. Ahn SS, Hwang SH, Jung SM, Lee SW, Park YB, Yun M, et al. Evaluation of Spleen Glucose Metabolism Using 18F-FDG PET/CT in Patients with Febrile Autoimmune Disease. J Nucl Med. 2017;58(3):507-13.

23. Lefebvre N, Argemi X, Meyer N, Mootien J, Douiri N, Sferrazza-Mandala S, et al. Clinical usefulness of 18F-FDG PET/CT for initial staging and assessment of treatment efficacy in patients with lymph node tuberculosis. Nucl Med Biol. 2017;50:17-24.

24. Alshammari A, Skoura E, Kazem N, Ashkanani R. Kikuchi Disease with Generalized Lymph Node, Spleen and Subcutaneous Involvement Detected by Fluorine-18-Fluorodeoxyglucose Positron Emission Tomography/Computed Tomography. Mol Imaging Radionucl Ther. 2016;25(2):102-6.

25. Ito K, Morooka M, Kubota K. Kikuchi disease: 18F-FDG positron emission tomography/computed tomography of lymph node uptake. Jpn J Radiol. 2010;28(1):15-9.

26. Sharma V, Rankin R. Fatal Kikuchi-like lymphadenitis associated with connective tissue disease: a report of two cases and review of the literature. Springerplus. 2015;4:167.

27. Zhuang H, Yu JQ, Alavi A. Applications of fluorodeoxyglucose-PET imaging in the detection of infection and inflammation and other benign disorders. Radiol Clin North Am. 
2005;43(1):121-34.

28. Meller J, Altenvoerde G, Munzel U, Jauho A, Behe M, Gratz S, et al. Fever of unknown origin: prospective comparison of [18F]FDG imaging with a double-head coincidence camera and gallium-67 citrate SPET. Eur J Nucl Med. 2000;27(11):1617-25.

29. de Porto AP, Lammers AJ, Bennink RJ, ten Berge IJ, Speelman P, Hoekstra JB. Assessment of splenic function. Eur J Clin Microbiol Infect Dis. 2010;29(12):1465-73.

30. Nam HY, Kim SJ, Kim IJ, Kim BS, Pak K, Kim K. The clinical implication and prediction of diffuse splenic FDG uptake during cancer surveillance. Clin Nucl Med. 2010;35(10):759-63.

\section{Figure legends}

Figure 1. Maximum intensity projection images of representative mild and severe KikuchiFujimoto disease patients with lesion segmentation. (a) A patient with severe Kikuchi-Fujimoto disease. Systemic increase in F18-FDG uptake in the spleen and lymph nodes is seen. (b) A patient with mild Kikuchi-Fujimoto disease. Lesser FDG-avid lymph nodes are seen.

\section{Figure 2. Receiver operating characteristic curve of metabolic parameters in Kikuchi-Fujimoto} disease. The area under the curve (AUC) for total lymph nodes, TLG, and spleen SUV mean are higher than that of total lymph nodes MTV.

$\mathrm{SUV}_{\text {mean }}$, mean standardized uptake value; MTV, metabolic tumor volume; TLG, total lesion glycolysis.

Table 1. Patient characteristics and results of univariate analysis for predicting severity of Kikuchi-Fujimoto disease

\begin{tabular}{lcccc}
\hline \multicolumn{1}{c}{ Characteristics } & $\begin{array}{c}\text { Total } \\
(\mathbf{N = 3 1})\end{array}$ & $\begin{array}{c}\text { Mild } \\
(\mathbf{N}=\mathbf{8})\end{array}$ & $\begin{array}{c}\text { Severe } \\
(\mathbf{N}=\mathbf{2 3})\end{array}$ & $\boldsymbol{P}$ - value \\
\hline Age (years) & $27.5 \pm 28$ & $38.0 \pm 29$ & $26.0 \pm 18$ & 0.038 \\
Sex (male, \%) & $13(41.9)$ & $3(37.5)$ & $10(43.5)$ & $>0.999$ \\
Lymphadenopathy, yes (\%) & & & & \\
$\quad$ Cervical & $18(58.1)$ & $5(62.5)$ & $13(56.5)$ & $>0.999$ \\
Axillary & $7(22.6)$ & $2(25.0)$ & $5(21.7)$ & $>0.999$
\end{tabular}


Mediastinal

Abdominopelvic

4(12.9)

1(12.5)

$0(0.0)$

1(4.3)

0.456

\section{Systemic symptoms}

Fever

Night sweat

Sore throat

Weight loss

Rash

Myalgia

Arthralgia

Nausea

Headache

\section{Laboratory findings}

WBC $\left(10^{3} / \mathrm{ul}\right)$
$\operatorname{CRP}(\mathrm{mg} / \mathrm{L})$
$\operatorname{ESR}(\mathrm{mm} / \mathrm{hr})$
$\mathrm{LDH}(\mathrm{IU} / \mathrm{L})$

\section{PET-CT parameters}

Total lymph nodes $\mathrm{SUV}_{\max }$

Total lymph nodes MTV

Total lymph nodes TLG

Spleen $\mathrm{SUV}_{\text {mean }}$

Spleen TLG
$30(96.8)$

7(22.6)

4(12.9)

$5(16.1)$

$8(25.8)$

7(22.6)

$5(16.1)$

4(12.9)

2(6.5)

$3.720 \pm 4.52$

$22.75 \pm 73.35$

$48.0 \pm 39.75$

$505.5 \pm 604$

$10.65 \pm 7.64$
$97.95 \pm 112.29$
$398.73 \pm 464.08$
$2.19 \pm 1.14$

$8.19 \pm 7.10$

0.214

$61.85 \pm 118.32$

$99.36 \pm 120.21$

0.104

$201.57 \pm 363.45$

$473.52 \pm 504.44$

0.024

$539.24 \pm 395.69$

$1.79 \pm 0.99$

$2.38 \pm 1.18$

0.058

$391.78 \pm 528.87 \quad 548.73 \pm 380.91$

0.162
$11.68 \pm 7.33$ 
Table 3. Associated factors for severe Kikuchi-Fujimoto disease using logistic regression model

\begin{tabular}{|c|c|c|c|c|}
\hline \multirow{2}{*}{ Variables } & \multicolumn{2}{|c|}{ Univariate } & \multicolumn{2}{|c|}{ Multivariable } \\
\hline & OR (95\% CI) & $P$ value & OR (95\% CI) & $P$ value \\
\hline Age & $0.956(0.910-1.004)$ & 0.073 & & \\
\hline Sex & & 0.768 & & \\
\hline Female & Reference & & & \\
\hline Male & $1.282(0.246-6.688)$ & & & \\
\hline \multicolumn{5}{|l|}{ Systemic symptoms } \\
\hline Fever duration & $1.212(0.941-1.560)$ & 0.137 & & \\
\hline Myalgia & $0.150(0.024-0.946)$ & 0.044 & $0.035(0.001-0.792)$ & 0.035 \\
\hline \multicolumn{5}{|l|}{ Laboratory findings } \\
\hline $\mathrm{CRP}(\geq 100 \mathrm{mg} / \mathrm{L})$ & $0.632(0.092-4.350)$ & 0.641 & & \\
\hline LDH $(\geq 1000 \mathrm{IU} / \mathrm{L})$ & $1.944(0.192-19.741)$ & 0.574 & & \\
\hline \multicolumn{5}{|l|}{ PET-CT parameters } \\
\hline Total lymph nodes $\mathrm{SUV}_{\max }(>9.27)$ & $8.500(1.335-54.127)$ & 0.023 & $24.734(1.323-462.407)$ & 0.032 \\
\hline Total lymph nodes MTV (> 34.72) & $6.667(1.057-42.065)$ & 0.044 & & \\
\hline Total lymph nodes TLG (> 429.99) & $10.889(1.140-103.977)$ & 0.038 & & \\
\hline Spleen $\mathrm{SUV}_{\text {mean }}(>1.79)$ & $11.111(1.701-72.564)$ & 0.012 & $37.770(1.769-806.583)$ & 0.020 \\
\hline Spleen TLG (> 296.06) & $10.500(1.412-78.059)$ & 0.022 & & \\
\hline Relapse & $1.050(0.093-11.824)$ & 0.968 & & \\
\hline Steroid use & $0.762(0.122-4.751)$ & 0.771 & & \\
\hline
\end{tabular}




\section{Figures}

(a)

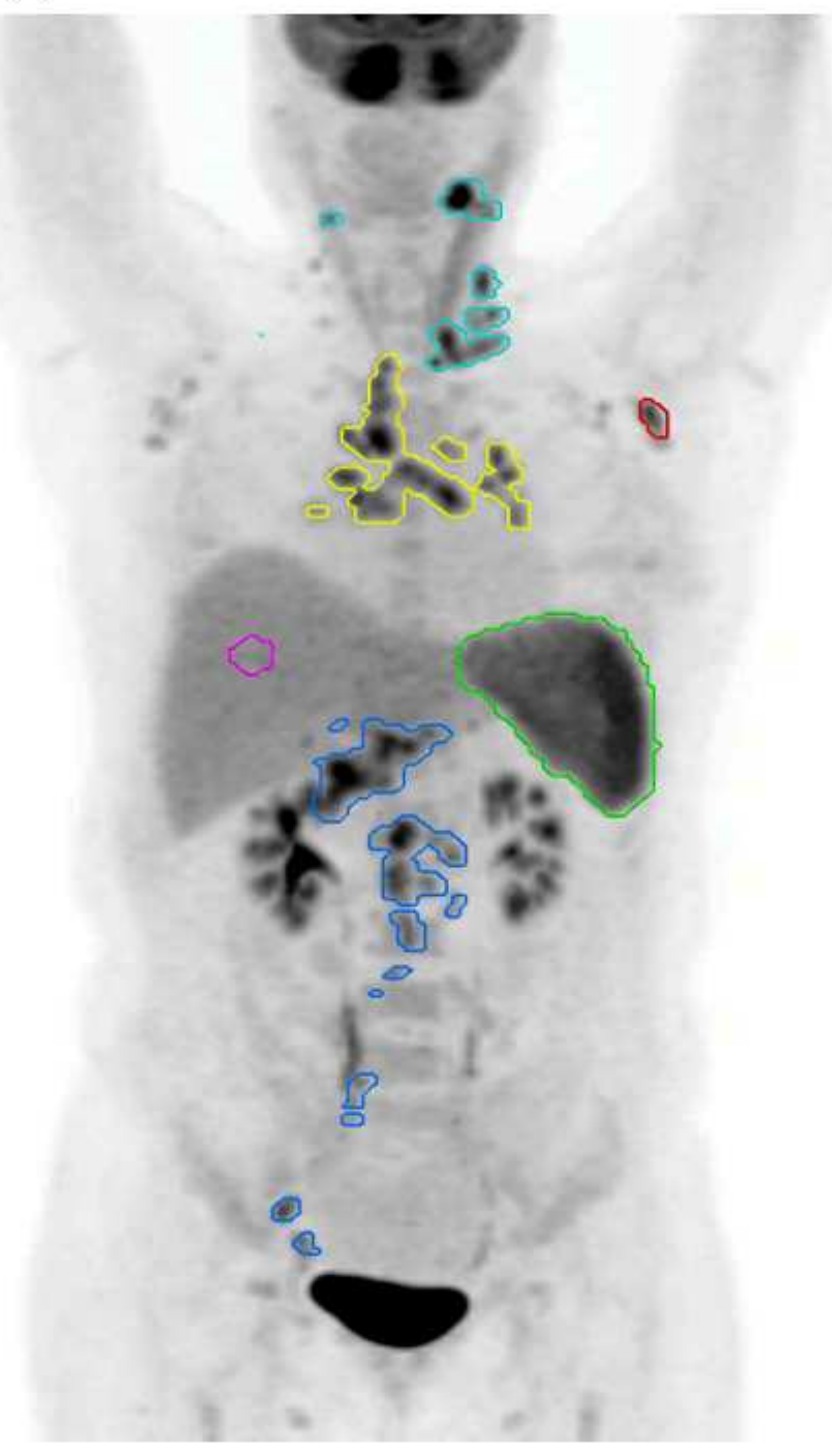

(b)

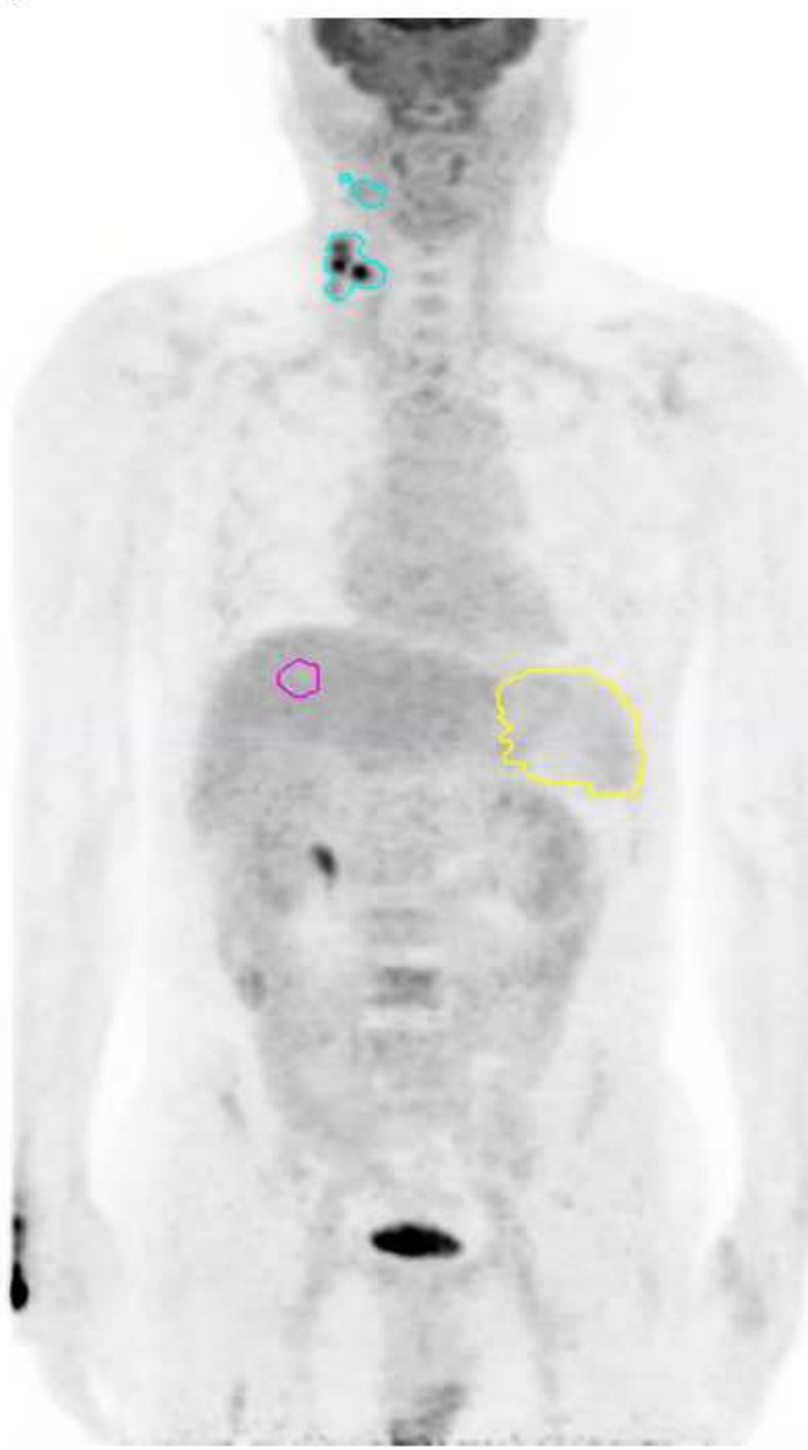

Figure 1. Maximum intensity projection (MIP) images of representative mild and severe Kikuchi-Fujimoto disease patient with lesion segmentation. (a) patient classified as severe Kikuchi-Fujimoto disease patient. Systemic increase in FDG uptake in the spleen and lymph nodes are seen. (b) patient classified with mild Kikuchi-Fujimoto disease. Fewer FDG-avid lymph nodes are seen.

\section{Figure 1}

Maximum intensity projection images of representative mild and severe Kikuchi-Fujimoto disease patients with lesion segmentation. (a) A patient with severe Kikuchi-Fujimoto disease. Systemic increase in F18-FDG uptake in the spleen and lymph nodes is seen. (b) A patient with mild Kikuchi-Fujimoto disease. Lesser FDG-avid lymph nodes are seen. 


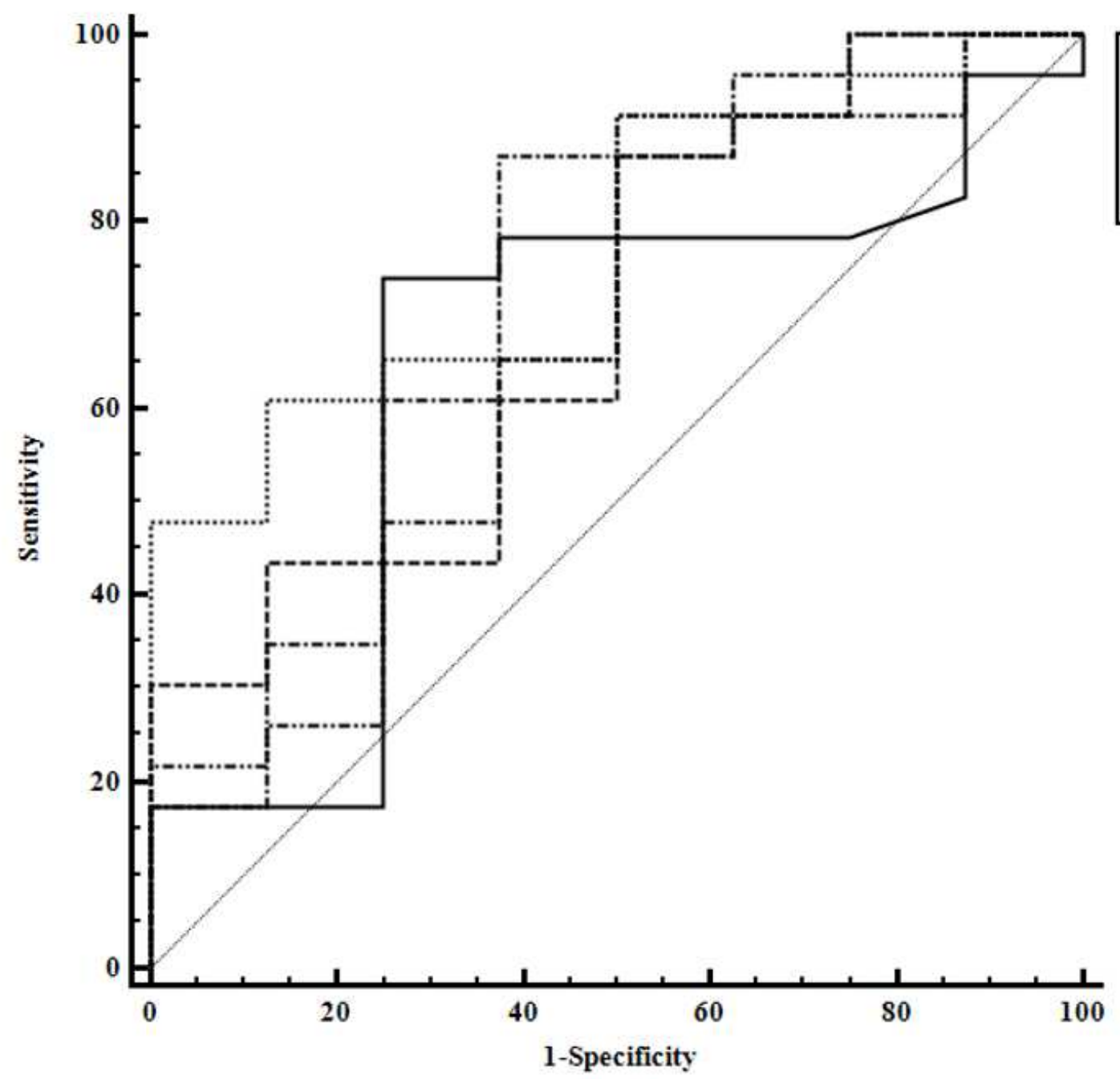

Figure 2. Receiver operating characteristic (ROC) curve of metabolic parameters in KikuchiFujimoto disease. The area under curve (AUC) for total lymph nodes TLG and spleen SUV mean $_{\text {are }}$

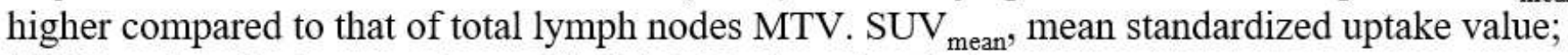
MTV, metabolic tumor volume; TLG, total lesion glycolysis.

Figure 2

Receiver operating characteristic curve of metabolic parameters in Kikuchi-Fujimoto disease. The area under the curve (AUC) for total lymph nodes, TLG, and spleen SUVmean are higher than that of total lymph nodes MTV. SUVmean, mean standardized uptake value; MTV, metabolic tumor volume; TLG, total lesion glycolysis. 